\title{
A theory-informed systematic review of clinicians' genetic testing practices
}

\author{
Jean L. Paul (1) ${ }^{1,2} \cdot$ Hanna Leslie ${ }^{1,2,3} \cdot$ Alison H. Trainer ${ }^{4,5} \cdot$ Clara Gaff $^{1,4,6}$
}

Received: 2 November 2017 / Revised: 13 April 2018 / Accepted: 8 May 2018 / Published online: 11 June 2018

(c) European Society of Human Genetics 2018

\begin{abstract}
This systematic literature review investigates factors impacting on clinicians' decisions to offer genetic tests in their practice, and maps them to a theoretical behaviour change framework. Better understanding of these factors will inform the design of effective interventions to integrate genomics tests into clinical care. We conducted a narrative synthesis of empirical research of medical specialists' perspectives on and experiences of offering genetic tests to their patients. This review was based upon the PRISMA statement and guidelines for reviewing qualitative research. Four electronic data sources were searchedMEDLINE, EMBASE, CINAHL, PubMed. Studies were independently assessed by two authors. Content analysis was applied to map the findings of included studies to a framework validated for behaviour and implementation research, the Theoretical Domains Framework (TDF). The TDF describes 14 factors known to influence behaviour and has been applied in diverse clinical settings to understand and/or modify health professional behaviour. Thirty-four studies published in 39 articles met inclusion and quality criteria. Most studies were published after 2011 (54\%), Northern American (82\%), quantitative in design (68\%) and addressed familial cancer genetic tests (53\%). Of the 14 TDF factors, 13 were identified. The three most common factors were: Environmental Context and Resources $(n=33)$, Beliefs about Consequences $(n=26)$, and Knowledge $(n=23)$. To support the adoption of genomic tests beyond specialist services, nuanced interventions targeting considerations beyond clinician education are needed. For instance, interventions addressing organisational constraints which may restrict clinicians' ability to offer genomic tests are required alongside those targeting factors intrinsic to the clinician.
\end{abstract}

Electronic supplementary material The online version of this article (https://doi.org/10.1038/s41431-018-0190-7) contains supplementary material, which is available to authorized users.

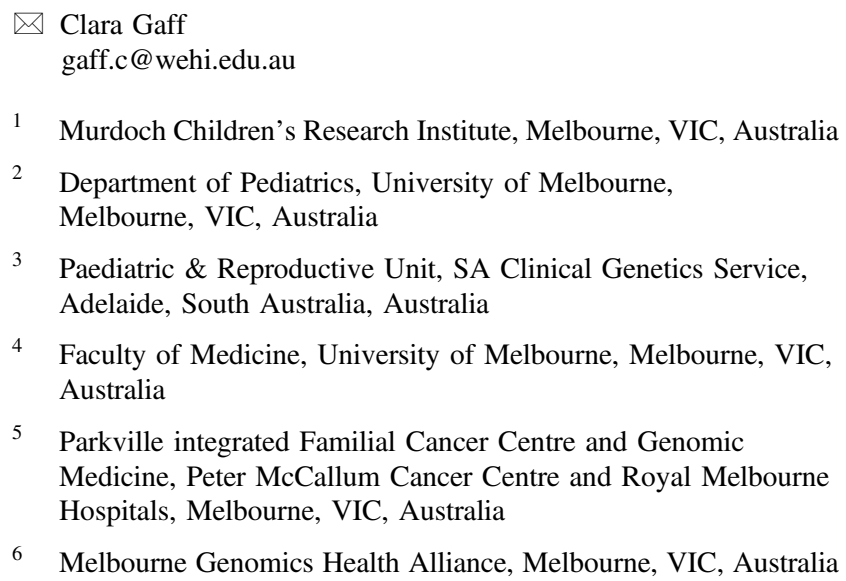

4 Faculty of Medicine, University of Melbourne, Melbourne, VIC, Australia

5 Parkville integrated Familial Cancer Centre and Genomic Medicine, Peter McCallum Cancer Centre and Royal Melbourne Hospitals, Melbourne, VIC, Australia

6 Melbourne Genomics Health Alliance, Melbourne, VIC, Australia

\section{Introduction}

Genomic technologies have significantly increased the breadth of genomic variation that can be accessed in a clinically relevant timeframe, and have potential to radically transform healthcare service provision across multiple, if not all clinical specialties [1]. While specialist genetic and cancer services are currently at the forefront of introducing genomic tests, it is likely that use of this testing will be applied in the 'mainstream' of other specialist healthcare over time. Early prospective evidence supports the costeffectiveness of genomic tests in specific contexts [2]. However, as with many clinical research findings, realising patient outcomes is dependent on clinicians adopting the test into usual practice, and integrating the resultant information appropriately into clinical management decisions.

Experts have identified numerous barriers to adoption of genomic tests, one of which is lack of clinician preparedness [3]. It is anticipated that specialists in clinical genetics would be early adopters of new genomic 
technologies where possible [4], but this may not be the case for other medical disciplines. Previous research assessing the introduction of single-gene tests described 'clinical inertia' [5] between test development and its integration into mainstream healthcare findings [6-8]. Concerns around the introduction of genomic tests echo these [5]. Health professionals remain underprepared to appropriately incorporate even single-gene genetic testing into their practice, let alone more extensive multigene panels and genomic sequencing [9-14]. This is important as over-use and under-use of genomic tests by medical practitioners could reduce benefits to patients and costeffectiveness of testing [15-17]. A common, but limited, reflex response to 'clinical inertia' has been to focus on educating clinicians to address a knowledge gap [6, 9]. Hamilton et al. investigated the successful implementation of genetic tests in the Veterans Health Administration system as a surrogate for genomic tests, finding that this relied on knowledgeable clinicians and organisationallevel facilitators [18]. Medical practitioners' decisionmaking regarding genetic or genomic testing in mainstream practice however, has not been systematically examined. Klitzman et al.s' observation remains pertinent; 'Many questions remain concerning whether, when and how physicians order genetic tests and what factors are involved in their decisions' [19].

As has been identified within the Cochrane Effective Practice and Organization of Care (EPOC) group [20-22], overcoming 'clinical inertia' to maximise appropriate use of testing requires deliberate, targeted interventions to optimise behaviour in the relevant clinical context. So, to effectively integrate genomic testing into mainstream medicine, factors which impact on medical practitioners' decisions to offer a genomic test must be systematically identified, then optimised [23]. Such an approach has been recommended by the UK's Chief Medical Officer in the 2016 annual report, Generation Genome [24], but a systematic review identified a lack of relevant research [23].

The Theoretical Domains Framework (TDF) provides a validated agnostic tool by which factors influencing clinicians' decisions to offer a genomic test can be systematically identified. Based on behaviour change theory, this framework categorises drivers and barriers of behaviour change into 14 key domains such as social influences, knowledge, skill and environmental context (Table 1) [25]. Three key aspects make it particularly suitable for research into clinician behaviour and decision-making around genomic testing. First, the TDF has been extensively used to investigate clinicians' practices and is thus well validated in this field. Second, it is agnostic to clinical specialty-an important consideration given the potential breadth of application of genomics in clinical practice. Finally, the TDF sits at the core of the Behaviour Change Wheel: developed by Michie et al. [26]; the Behaviour Change Wheel maps the 14 TDF factors onto a clinician's capability, opportunity and motivation to offer a test, and then identifies evidence-based intervention mechanisms targeted to these areas. Through the Behaviour Change Wheel, the TDF not only provides a direct framework with which to identify barriers and facilitators of genomic test integration, but also indirectly identifies plausible, evidencebased interventions to optimise its integration.

\section{Rationale and objective}

Interventions to support adoption of current genomic tests will be most effective if two conditions are met. First, they are informed by a comprehensive understanding of factors influencing medical practitioners' use of single-gene testing. Second, they are based upon a well-established, practical framework designed to optimise practitioner behaviour [23]. We sought to identify key, modifiable mediators of behaviour change which can guide the adoption of new technologies within the healthcare system, applying the Theoretical Domains Framework. This review systematically examines existing published literature to identify factors that impact upon medical specialists' decisions to offer single-gene tests in clinical practice. To address the adoption of genomics beyond specialist genetic services, we focus on the use of tests by other medical specialists caring for people who either have a clinical condition or are at high risk of a hereditary disease.

\section{Methods}

\section{Design}

Guided by: the PRISMA statement for reporting systematic reviews [27] and Economic and Social Research Council UK guidelines for reviews incorporating qualitative research [28].

\section{Eligibility criteria}

Studies were eligible for inclusion if they satisfied the following criteria (developed by HL, AT, CG):

- original primary peer-reviewed research;

- obtained evidence about factors which impacted on medical specialists' decisions to offer genetic tests;

- and genetic test was germline and currently available for the patients' clinical care.

Studies were excluded if they: 


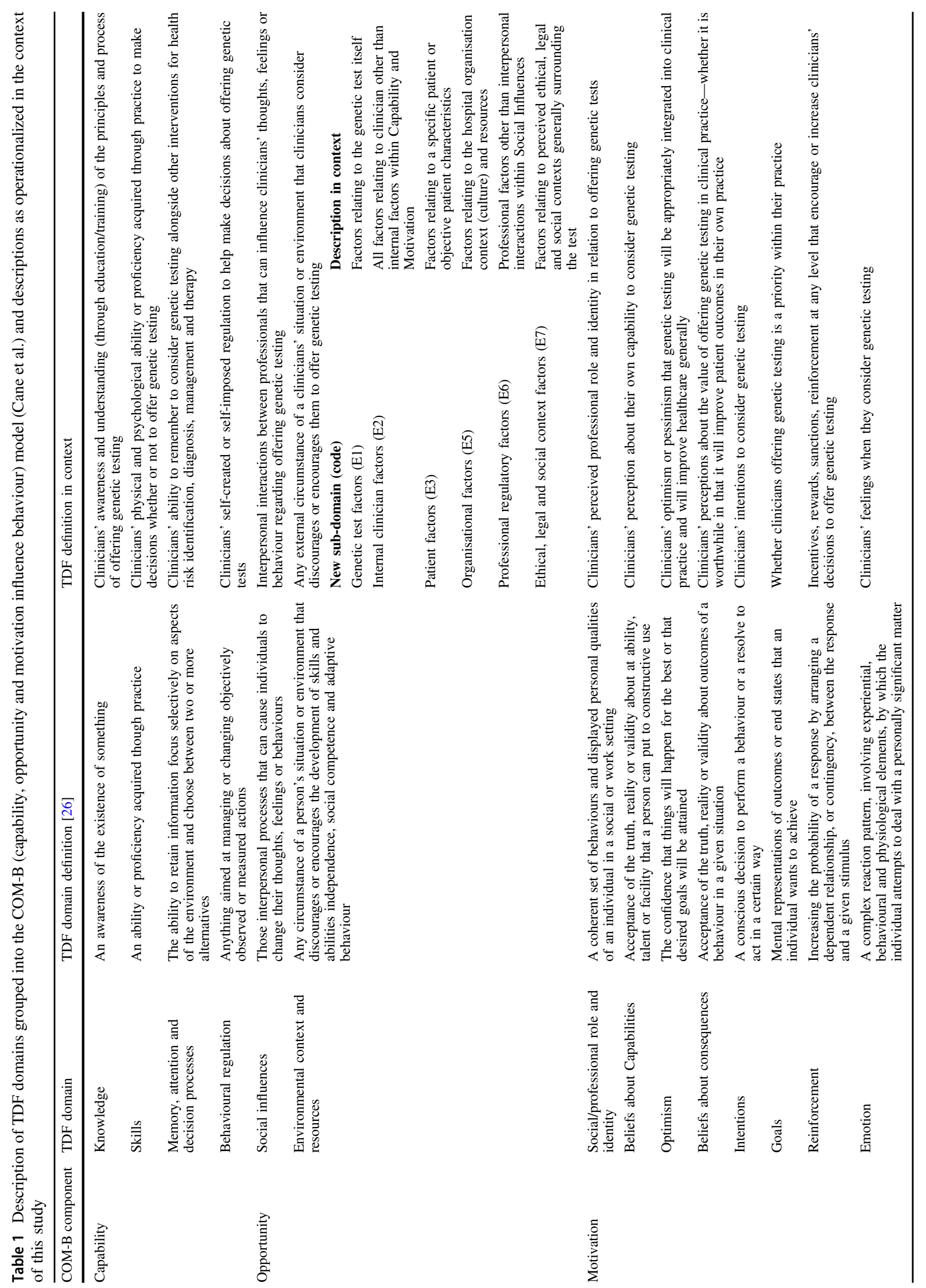


Table 2 Electronic search results

\begin{tabular}{lllll}
\hline Search term ${ }^{\mathrm{a}}$ & \multicolumn{2}{l}{$\begin{array}{l}\text { 'Genetic testing/', 'Physician's Practice Patterns/' and 'Health } \\
\text { Knowledge, Attitudes, Practice/'. }\end{array}$} \\
\cline { 2 - 5 } Electronic databases searched & Date searched & Search type & Years & Result \\
\hline MEDLINE & $29 / 09 / 2015$ & MeSH Topics & All & 339 \\
Embase & $29 / 09 / 2015$ & MeSH Topics & All & 418 \\
CINAHL & $29 / 09 / 2015$ & MeSH Topics & All & 263 \\
PubMed & $29 / 09 / 2015$ & Keyword & All & 146 \\
& & & TOTAL & 1166 \\
Duplicates removed: & & & TOTAL & $\mathbf{1 0 2 6}$ \\
\hline
\end{tabular}

${ }^{\mathrm{a}}$ Full search term available upon request
- were not published in English;

- involved a clinical scenario for a test that did not exist;

- concerned return of results or family communication;

- involved only medical (clinical) geneticists;

- or concerned a prenatal, population, somatic or direct-toconsumer (DTC) genetic test.

Prenatal or preimplantation tests were excluded as this review focuses on medical specialists' decision-making behaviour regarding genetic tests used to determine management of that person. Population screening programmes were also excluded as no clinician-based decision-making is required.

\section{Search}

Four electronic databases were selected using a detailed search strategy developed for MEDLINE and adapted for other databases (Table 2).

\section{Study selection}

Studies were assessed for inclusion by two reviewers (a third used if discordance was encountered) as follows: exclusion based on title, abstract and screening the remaining full-texts for inclusion. Reference lists were hand-searched during study screening and selection.

\section{Data extraction}

Identifying information was extracted: first author, publication year, country of origin and study design (quantitative, qualitative or mixed methods). The following was also collected: principal objective, clinician population (including clinician type and sample size), response rate, clinical domain (e.g. oncology, paediatrics or multiple domains), indication for test (e.g. predictive or diagnostic) and genetic testing platform (traditional single-gene testing or whole-genome testing). Using deductive content analysis [29], study findings were mapped in a non-mutually exclusive manner to the 14 TDF domains (Table 1) and further sub-grouped by clinician's capability, opportunity and motivation according to the Behaviour Change Wheel framework. Findings which were acting on the medical specialists' decision to offer genetic testing were investigated and mapped to the TDF. Coding was conducted by two authors (HL and JP) and discussed and amended among all authors. Findings could be coded to multiple domains. The QSR International software NVivo10 facilitated data management and storage [30].

\section{Risk of bias in individual studies}

Each article was independently evaluated for methodological quality (HL, JP). Due to heterogeneity in study design and analytical approaches, a quality appraisal tool, based upon QualSyst [31] as described by Paul and colleagues [32], was adapted and applied to best assess the elements relevant to the aims of the review and study design. Briefly, all studies were assessed using eight standard questions plus additional questions specific to the underlying methodology: qualitative or quantitative. The total study score was divided by the total possible score. The questions applied and scoring scheme are provided in Supplementary Table 1. Only those with an average score above 0.70 were included.

\section{Synthesis of results}

A meta-analysis was not feasible due to study heterogeneity in primary aims, design, method and populations. A narrative synthesis [28] was therefore conducted to allow for comprehensive description and interpretation. Findings are presented in descriptive frequencies of TDF domains and narrative form. As a finding may map to more than one domain, its discussion in regard to one domain below does not exclude its inclusion in other domains. The unit of analysis, or 'study', was the series of published articles reported from a single data set. 


\section{Results}

\section{Search outcome}

From the total articles retrieved (Table 2), most were removed at title or abstract stage, while only five were included through reference-searching. Eight were excluded based upon quality score, leaving a total of 39 articles from 34 studies [15, 19, 33-69] (Fig. 1).

\section{Characteristics of included studies}

Table 3 summarises characteristics of the 34 studies included. The majority were published within the last five years (54\%), originated from Northern America (82\%), and were quantitative in design (68\%). Collectively, they spanned many medical disciplines and included a mixture of medical specialists (53\%), although were highly enriched for studies based within oncology (53\%). A wide spectrum of genetic tests was explored, the majority being predictive genetic tests $(65 \%)$.

\section{Overview of study designs and quality}

Most studies investigated clinicians' knowledge, attitudes or beliefs about genetics and testing in clinical practice (Table 4, Study Details), using exploratory cross-sectional surveys and descriptive statistics. Twenty-five explored perspectives and knowledge of traditional diagnostic genetic testing techniques, while seven examined pharmacogenetics to guide drug dosage in various health conditions [37, 46, 52, 56, 60, 61, 64]. Only two studies explored use of newer genetic tests such as microarray testing $[35,58]$. No studies examined the use of genomic tests (multigene panel, exome or genome sequencing). Many studies had low response rates, three being below $10 \%$ [39, 47, 59, 61]. Few provided clear justifications for their methodological approach and most lacked an explicit theoretical model in their design; only one qualitative study described using 'grounded theory' [35]. Studies generally included limited detail regarding approaches to data collection, clinicians' demographics and study setting.

\section{Overview of TDF factors identified}

Thirteen of the 14 (TDF) factors (Table 1, overview of TDF factors) were identified from studies' findings as impacting clinicians' ability to offer genetic testing; only Behavioural Regulation was not represented (Fig. 2). The most commonly identified factors were: Environmental Context and Resources $(n=33)$, Beliefs about Consequences $(n=26)$ and Knowledge $(n=23)$, elaborated below.
Clinicians consider numerous 'environmental context and resource' parameters $(n=33)$

This domain comprises six further components, based upon healthcare practice-change research $[70,71]$ (see Table 1 for full descriptions). All six sub-domains were identified (Fig. 2), patient factors being most common $(n=26)$, followed by ethical, legal and social-based factors $(n=25)$. Clinicians' characteristics were identified least $(n=14)$. In order of frequency, environmental and resources constructs identified were:

Patient factors Patient factors relates to both tangible patient characteristics and clinicians' considerations of an individual patient. Clinicians considered patient characteristics, such as age [33], gender [41], family history [44, 50, 57], or particular health condition and indication for testing [33, 37, 67, 68], when deciding if testing should be offered. Across several studies, patient interest and patients' 'escalating expectations' [36] acted as facilitators for clinicians considering offering genetic testing [36, 41, 42, 56, 63, 66-68], even when contrary to guidelines [15]. In contrast, clinician-based perceptions that a specific patient may lack the ability to pay or experience a negative reaction to the test outcome, acted as barriers to offering genetic testing [38, 40].

Ethical, legal and social context factors Clinicians were concerned about potential non-medical implications of testing, including privacy [60], health insurance $[34,38,60]$ (U.S. studies) and equity of access in terms of test costs [38, 49, 51, 52]. In addition to consideration of impacts relating to an individual patient (included as 'patient factors' above), it was evident that clinicians' general perceptions of the potential impact of testing may influence their decisions, for example the potential 'psychological stress' of an incidental finding [58] or the potential for a reduction in stigma [63]. This may result from clinicians' previously perceived negative experiences of genetic testing for their patients, specifically with predictive testing for Huntington [36] or Alzheimer disease when there is no available treatment [67]. Clinicians were less willing to offer testing if they believed testing might produce falsepositive results [15].

Organisational resources Clinicians reported a lack of organisational resources at a health-service level as a barrier to testing [39], particularly, limited time for considering testing within their current practice $[35,51,60]$, especially if the testing process was not clear $[48,67,68]$ or not the standard of care at their practice [39], and extra time required for professional development [51, 58]. Long turnaround time for results also acted as a barrier to offering a genetic test $[43,46]$. Other perceived barriers included: 


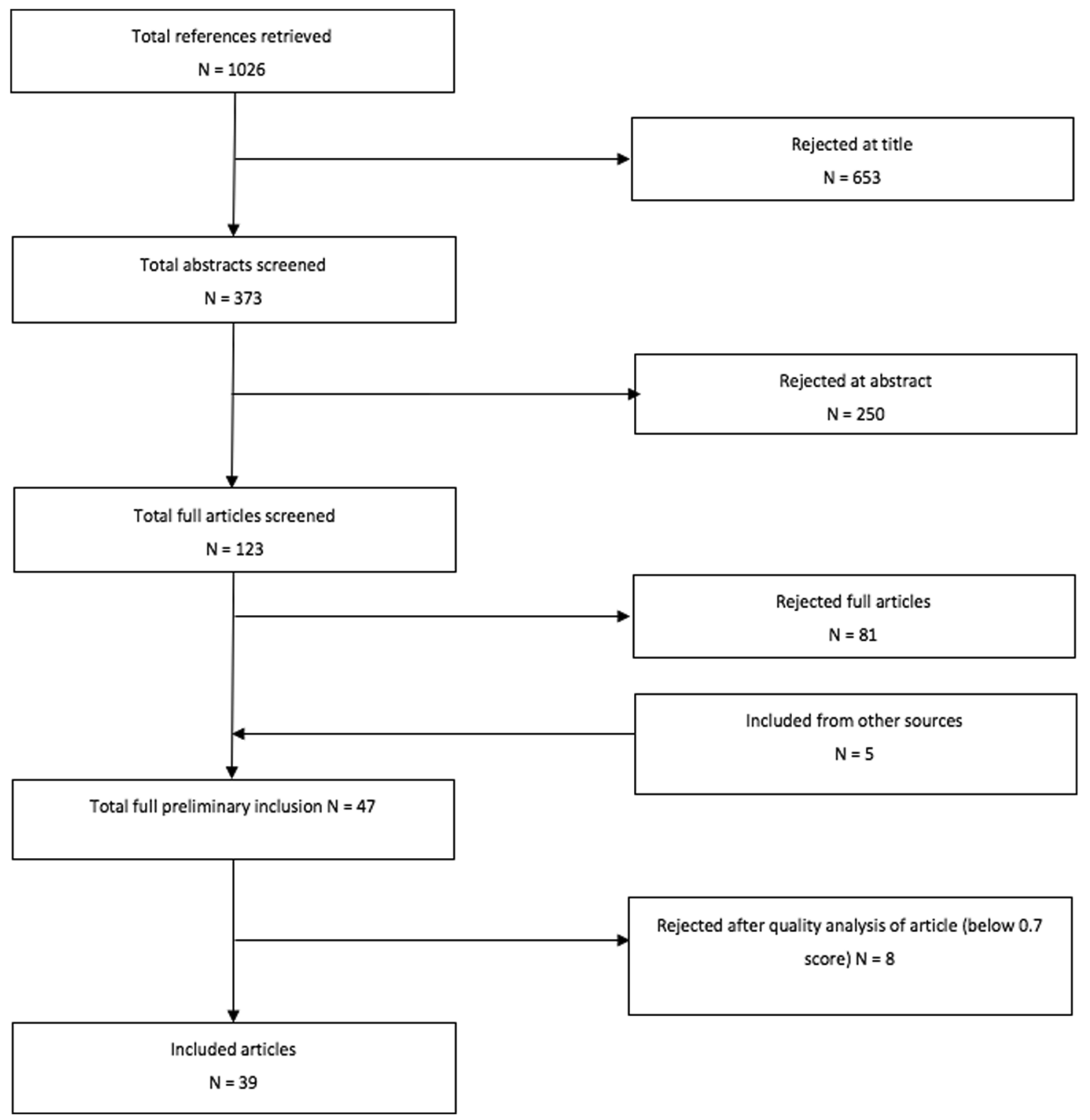

Fig. 1 PRISMA flow demonstrating study selection

seeing few cases requiring testing [17]; or if the hospital was based in a regional location [40]. Alternatively, genetic counsellor availability or a close working relationship with genetic specialists and testing laboratories $[48,58,61]$ could act as a facilitator [34]. Having an organisational policy for access to consent forms, educational literature for families [58] and requesting tests [50] could also influence clinicians' decisions to offer testing.

Genetic test factors Perceptions of clinical utility were a positive influence on clinicians' decisions $[15,37,43,56$, $67,68]$, while novelty and complexity of the test could act as a barrier $[36,37,51]$. Similarly, clinicians were hesitant to consider tests where medical implications of results were uncertain $[37,58]$, or if predictive or prognostic value of the test was perceived as uncertain $[36,51,52,63,69]$.

Professional regulatory factors Many studies identified that clinicians reported being highly influenced by professional guidelines $[34,37,39,40,50,56,60,65]$. If genetic testing was new or unfamiliar, clinicians believed that guidelines were not always complete [63] or available $[38,51]$, and they also perceived there to be a lack of (access to) relevant training [51, 53], and literature [46]. Where available, resources such as professional development and training were considered helpful when considering genetic testing [38, 41]. 
Table 3 Study characteristics

\begin{tabular}{|c|c|}
\hline Publication date & $N(\%$ of 39 articles $)$ \\
\hline$\leq 2000$ & $4(10 \%)$ \\
\hline $2001-2005$ & $8(21 \%)$ \\
\hline 2006-2010 & $6(15 \%)$ \\
\hline 2011-2015 & $21(54 \%)$ \\
\hline Country of origin & $N(\%$ of 34 studies $)$ \\
\hline North America & $28(82 \%)$ \\
\hline Europe & $4(12 \%)$ \\
\hline Australasia & $1(3 \%)$ \\
\hline Asia & $1(3 \%)$ \\
\hline Study design & $N$ (34 studies) \\
\hline Quantitative & $23(68 \%)$ \\
\hline Qualitative & $10(29 \%)$ \\
\hline Mixed methods & $1(3 \%)$ \\
\hline Clinician population $^{\mathrm{a}}$ & $N$ (34 studies) \\
\hline Mixed clinical population & $18(53 \%)$ \\
\hline Obstetricians/gynaecologists & $11(32 \%)$ \\
\hline Internists & $10(29 \%)$ \\
\hline Oncologists & $9(27 \%)$ \\
\hline Family physicians/GPs & $9(27 \%)$ \\
\hline Surgeons & $8(24 \%)$ \\
\hline Paediatricians & $5(15 \%)$ \\
\hline Neurologists & $3(9 \%)$ \\
\hline Psychiatrists & $3(9 \%)$ \\
\hline Not specified in study & $2(6 \%)$ \\
\hline $\begin{array}{l}\text { Other (including urologists, endocrinologists, } \\
\text { geriatricians, cardiologists) }\end{array}$ & $6(18 \%)$ \\
\hline Clinical domain ${ }^{a}$ & $N$ (34 studies) \\
\hline Oncology & $18(53 \%)$ \\
\hline Multiple clinical domains & $11(32 \%)$ \\
\hline Paediatrics & $6(18 \%)$ \\
\hline Neurology & $5(15 \%)$ \\
\hline Pre-pregnancy care & $5(15 \%)$ \\
\hline $\begin{array}{l}\text { Other (including psychiatry, haematology, } \\
\text { endocrinology, immunology) }\end{array}$ & $10(29 \%)$ \\
\hline Indication for test ${ }^{a}$ & $N$ (34 studies) \\
\hline Predictive & $22(65 \%)$ \\
\hline Diagnostic & $9(27 \%)$ \\
\hline Various indications & $8(24 \%)$ \\
\hline Pharmacogenetic & $7(21 \%)$ \\
\hline Carrier status & $4(12 \%)$ \\
\hline
\end{tabular}

${ }^{\mathrm{a}}$ Categories not mutually exclusive

Clinician factors The reported clinician-based parameters associated with requesting genetic tests were: (1) being an oncologist [15, 66]; (2) clinicians in U.S. hospitals who had completed their training in the United States [38]; (3) greater length of time since graduation [38]; and (4) working in a larger urban setting [38]. Parameters such as: clinician age; importance of religious values on decisionmaking, and personal experience with a genetic condition showed no associations.

\section{Beliefs about potential consequences when deciding to offer testing $(n=26)$}

The second most frequently identified domain related to clinicians' perceived value of offering genetic testing within their clinical practice. This intersects with environmental factors pertaining to ethical, legal and social considerations, with medical or clinical consequences placed in this code. Overall, clinicians had positive attitudes towards offering genetic tests, believing it could improve their patients' health. This acted as a facilitator to offering genetic testing. This perception varied based upon the type of test, the health condition and the availability of other tools for risk assessment, management or treatment.

Clinicians reported requesting genetic tests with the belief that results would guide future treatment, screening or management options [35, 38, 45, 52, 56, 61], including reproductive information [33]. They requested tests when they perceived it would reduce 'uncertainty' [43, 65], even when contrary to guidelines, for example clinicians considering breast cancer susceptibility testing in young [43] and/or unaffected relatives [55], regardless of uncertain results identified in the proband [57]. Conversely, clinicians did not consider genetic tests when they perceived such tests would not be relevant to their patients [38, 39], or did not believe results would change treatment [44, 52, 69]. Some clinicians were concerned about potential consequences of tests perceived as 'too new' [54, 63], believing a clear benefit had not yet been proven [37].

\section{Knowledge about genetics, testing processes and guidelines is varied $(n=23)$}

Clinician knowledge was assessed in studies across two main areas: (1) the genetic condition and test; and (2) clinical guidelines and test-requesting. Most studies which addressed these areas were quantitative in design and involved surveys. Overall, clinicians had good knowledge of basic genetics associated with conditions and an awareness of an appropriate genetic test [39, 40, 47, 55, 56, 61, 63]. However, there were significant differences in knowledge about different tests [52] and result types [58]. Knowledge was also found to be related to clinical indication [33], and clinician gender [64]. Several studies reported clinicians rated their knowledge of risk estimates and genetics as low $[15,50,63]$, clinicians believing they lacked sufficient knowledge to perform pre-test or post-test genetic counselling [34]. Clinicians' awareness and knowledge of current available guidelines was variable [40, 41, 57, 68]. They 


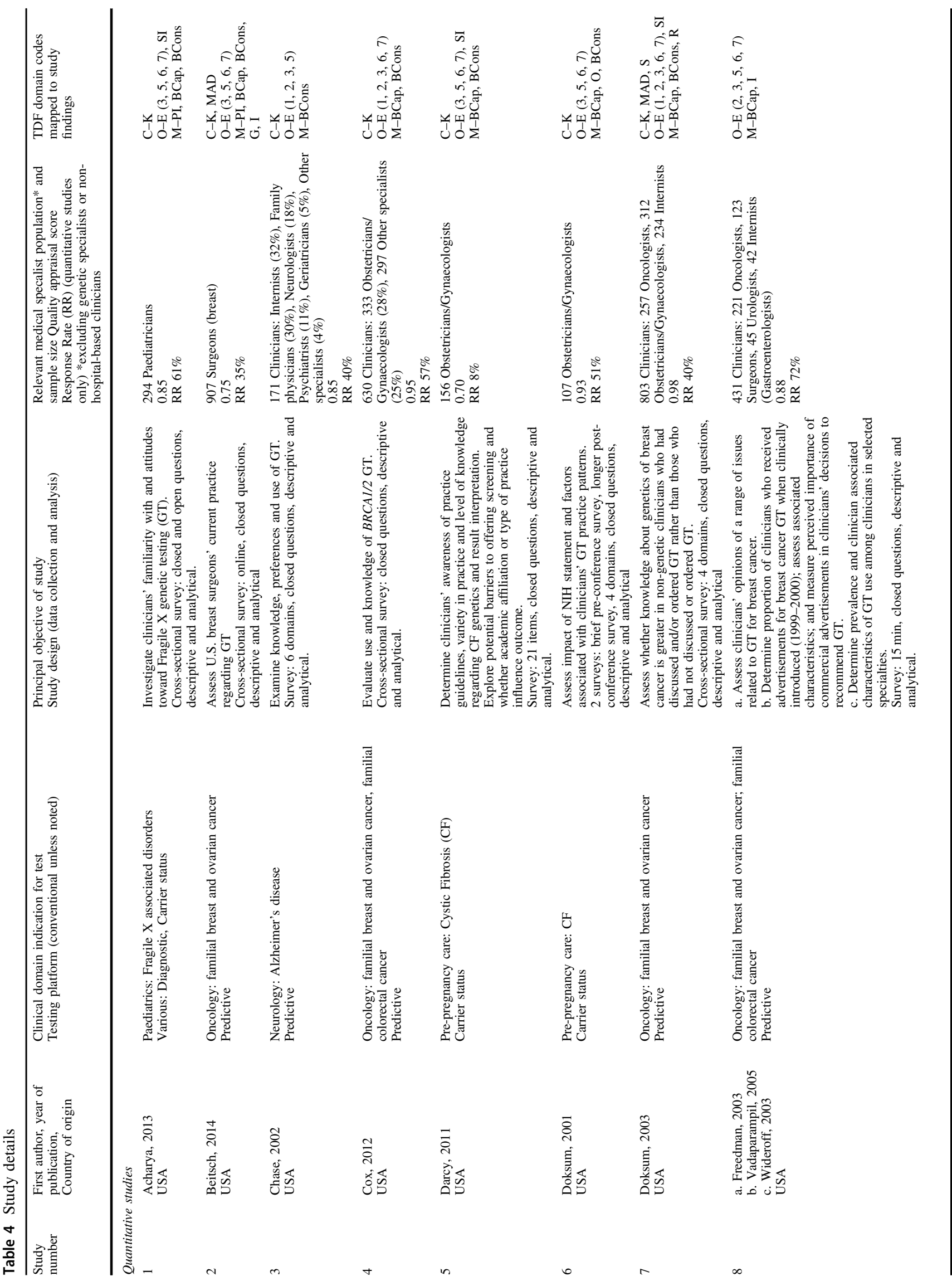




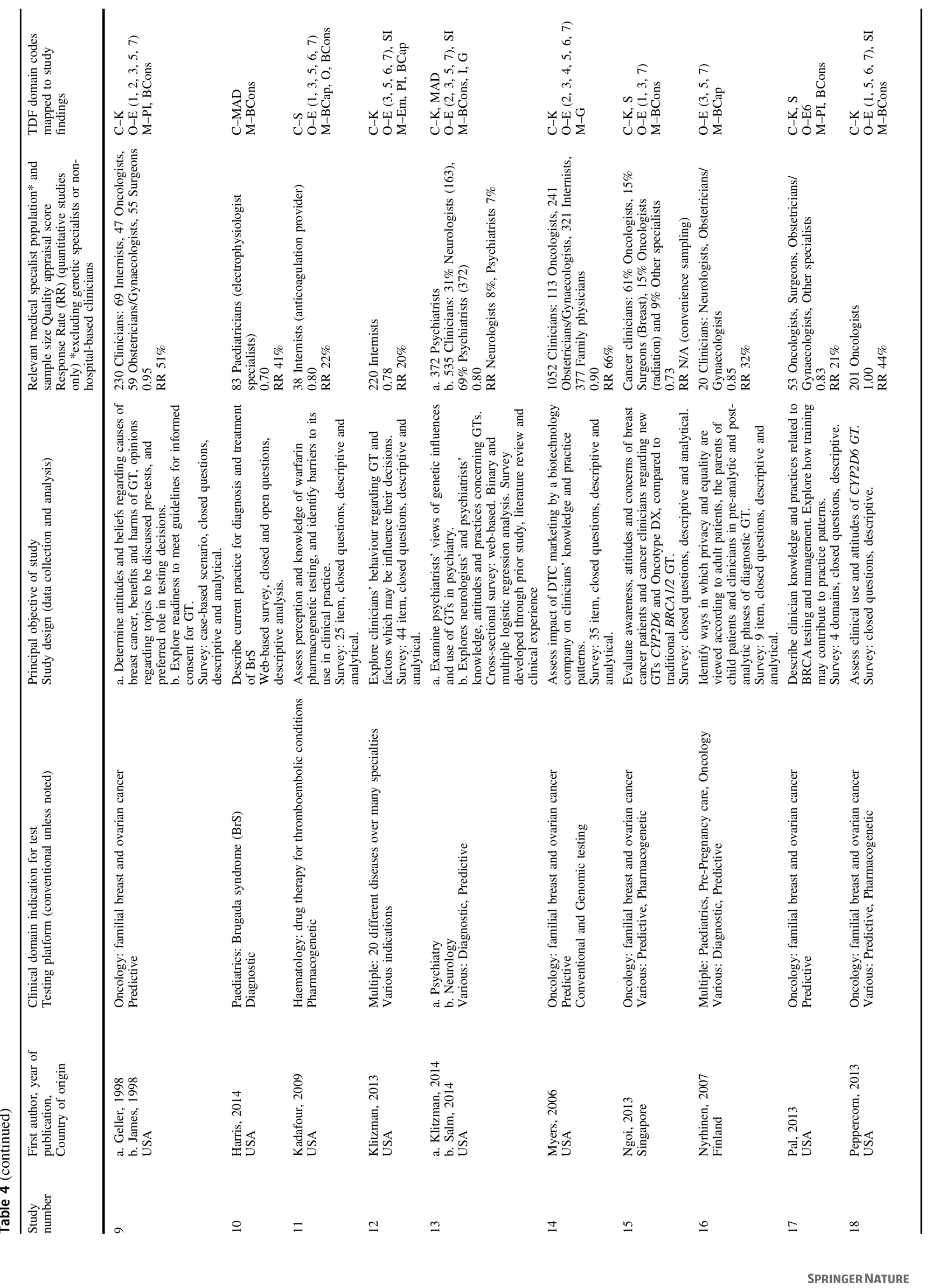




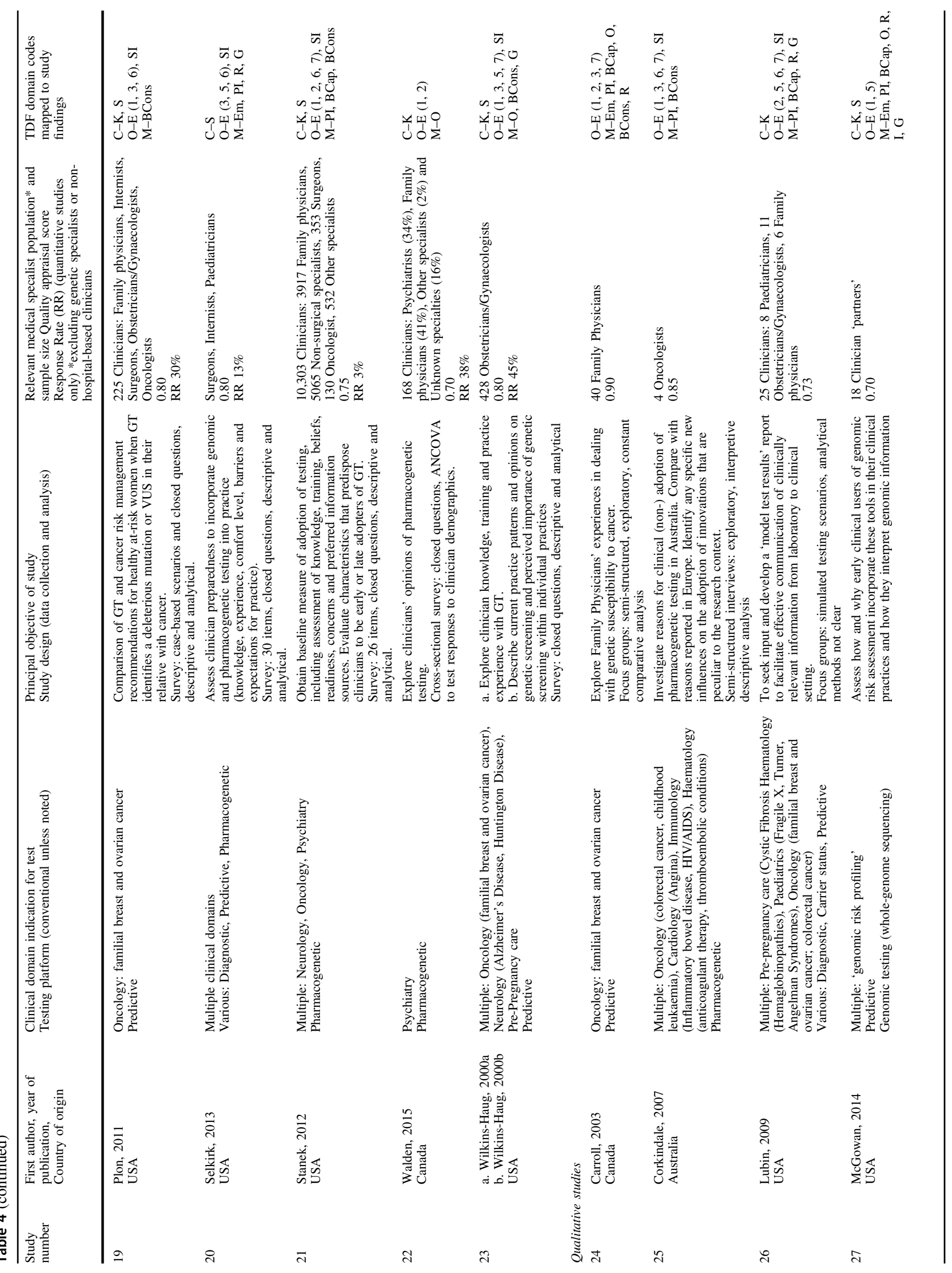




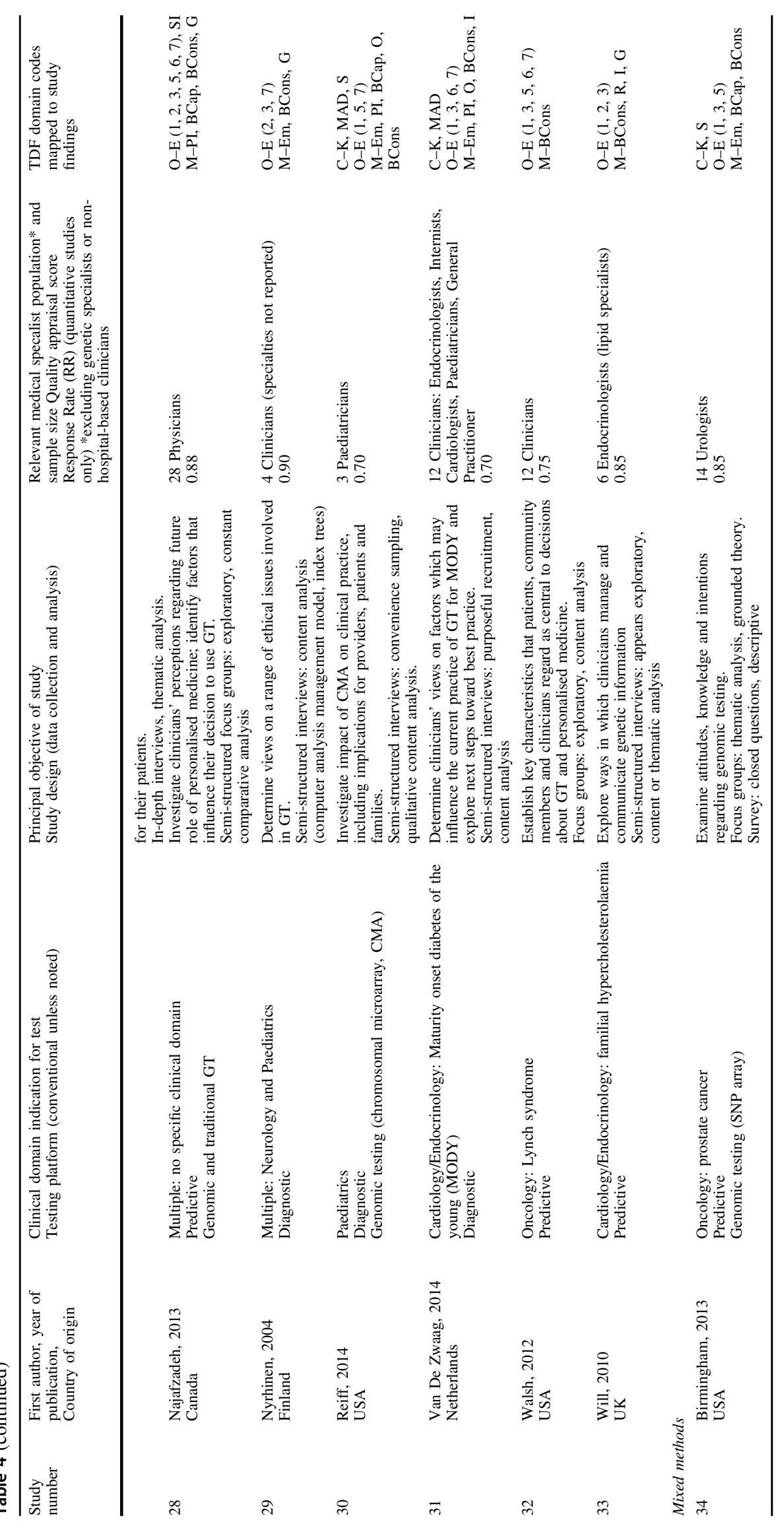


Fig. 2 Representation of TDF factors identified in studies $(n=$ 34)

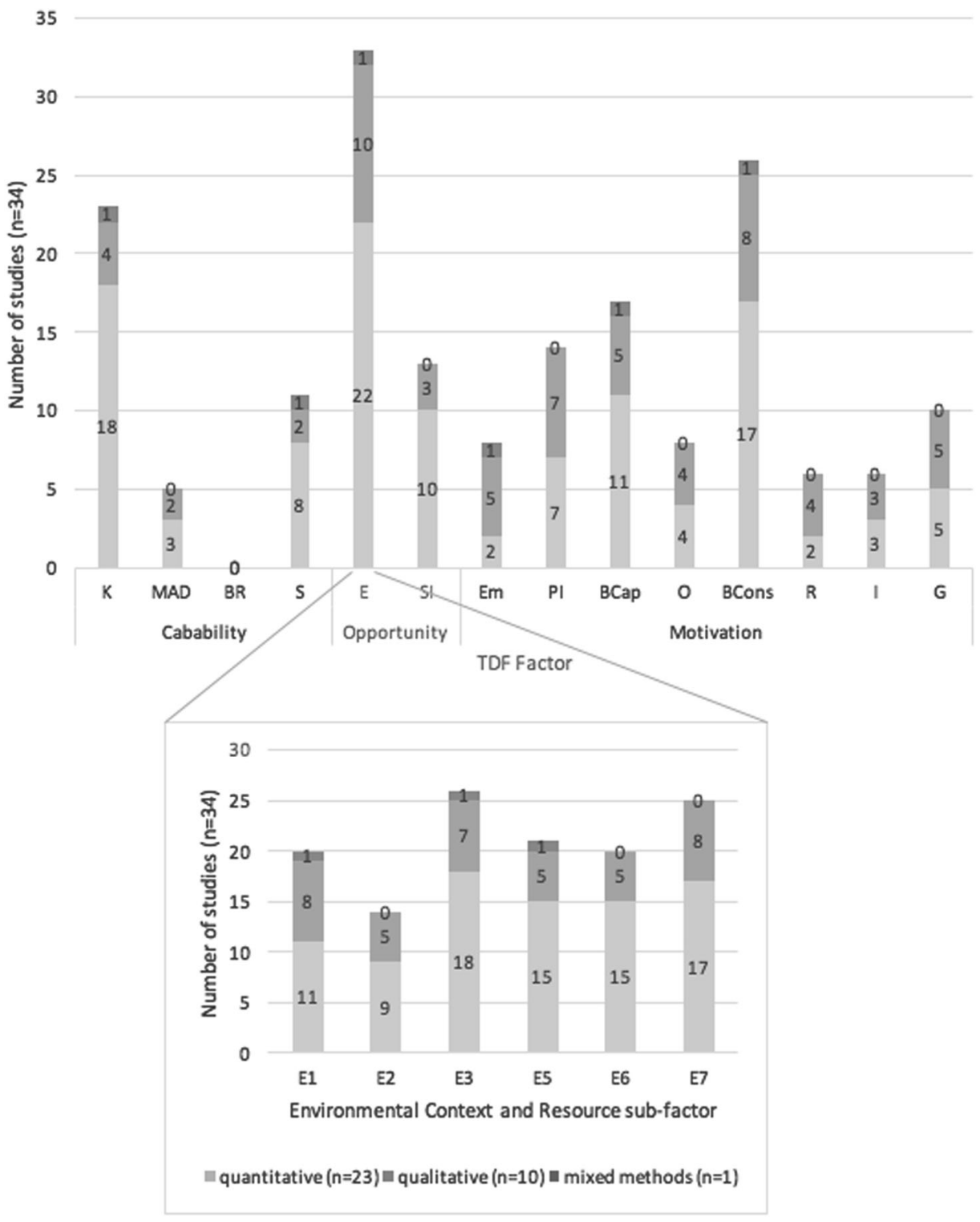

K- Knowledge; MAD - Memory, Attention and Decision Processes, 8R-8ehavioural Regulation, S-Skills, EEnvironmental Context and Resources (E1 - Genetic test factors, E2 - Internal clinician factors, E3 - Patient factors, ES Organizational factors, E6 - Professional regulatory factors, E7 - Ethical, legal, \& social context factors), SI - Social Influences, Em - Emotion, PI - Social/Professional Role and Identity, BCap - Beliefs about Capabilities, 0 - Optimism, BCons - Beliefs about Consequences, R - Reinforcement, I-Intensions, G - Goals often did not know the process for requesting genetic testing [48, 61]. However, other findings also revealed that clinicians had a good understanding of appropriate clinical use [35, 68]. Only two studies investigated relationships between knowledge and test-requesting behaviour [19, 41]. Klitzman et al.'s study found knowledge of the requesting process was a facilitator of testing [19] but did not investigate if testing was provided appropriately. Doksum and colleagues found that perfect knowledge scores were associated with discussing or requesting $B R C A 1 / 2$ genetic testing only for oncologists and not other disciplines within their study [41].

\section{Discussion}

This review identifies a complex network of factors which impact on clinicians' self-reported decisions to offer patients a genetic test. We found clinicians' decisions to offer testing is a nuanced process influenced by 13 parameters, nearly all of those known to influence behaviour change. Although behavioural regulation (that is, anything which clinicians do to adjust their own behaviour) was the only TDF factor not identified, this likely reflects the nature of the studies examined, rather than the factor's relevance. Our findings suggest that for effective adoption of genomic 
information into healthcare, a multi-level approach targeting institutional change as well as individuals will be required.

Much of the literature identified in this systematic review proposed educating clinicians as a means by which to change their practice in genetics $[15,19,33,34,36-42,46-48,50$, $51,53,56,57,60,61,63,67,68]$-indeed it is usually the only strategy proposed. Strikingly, none of these papers provided evidence for education as a means to change clinician behaviour. We did not identify any studies within the parameters of this systematic review that directly examined the effects of education on genetic testing requests. Furthermore, the studies did not draw on clinician behaviour change frameworks. This is most likely because the description of clinicians' test request patterns was usually only a minor element of larger studies. In fact, most studies only investigated clinician knowledge and opinions with no consideration given to alternative factors known to affect clinician behaviour. Interestingly, a systematic review to identify effective knowledge translation interventions in genetic testing found the majority focus on patient decision making, while evidence in other clinical areas indicates targeting both the health professional and the patient may be most effective [72].

Our findings support the principle that a clinician's decision to offer a genomic test will be a complex process resulting from an interplay of factors [23]. However, the studies identified in this review did not seek to explain clinician practice patterns or behaviours, looking instead at attitudes and potential associations between clinician characteristics and use of tests. This is an important gap in the literature. The offer of a new test may be a novel behaviour for the clinician, but it occurs in the context of decisions that the clinician already makes about the patient's management. Decisions about genomic testing could be seen in this light as a modification of an existing decision-making process, rather than being an entirely new decision. However, studies examined the decision to offer a genetic test in isolation from clinicians' usual patient care decision-making. There is a need for theoretically informed research studies specifically designed to make explicit the nature of these decisions.

Not all TDF factors identified are within clinician control. We found that organisational constraints had a significant impact on the offer of a test, suggesting institutional-based restructuring is necessary to support adoption. In view of this, it is significant that there was a total absence of studies identified which applied implementation science methodology. This scientific framework pragmatically and rigorously examines processes and services to determine how they may be optimised to improve a specific outcome. A roundtable discussion in the US recently advocated for an implementation science approach to genomic healthcare delivery [5].
It is tempting to offer suggestions of specific interventions based on the three most commonly identified factorsclinician knowledge, belief about consequences, and environmental context and resources. However, there is a paucity of high quality studies identified in this review to currently support the effectiveness of any intervention. Furthermore, the frequency of factors identified in this review is likely to relate to the design and purpose of the studies identified in this review, rather than their relative importance.

There is an inherent assumption in our systematic review that the provision of genomic tests for medical care will mirror that of genetic tests for hereditary disease, with primary care practitioners continuing to refer to other practitioners for both testing and specialised medical management of people with a condition. It is possible that genomic testing will be more widely used in primary care, particularly to predict the risk of disease in healthy people at population risk. However, the findings of our review are not applicable to that scenario, which is already being explored in pilot trials [73]. Nonetheless, we encourage research designed to specifically investigate primary care providers clinical decision-making and use of frameworks such as the TDF to enable insights into similarities and differences in decision-making across disciplines and clinical scenarios.

Many limitations arise from studies identified in this review. There were no longitudinal studies and most were largely reactive to the introduction of a genetic test. They therefore do not provide insights into changes that may occur over time, for example as clinicians become more familiar with tests or as costs decrease. Response rates were generally low, which was attributed in some cases to lack of interest by clinicians and suggests a possible bias in results. Findings from these studies may have over-estimated clinician knowledge and positive attitudes, as less interest in testing has been associated with lower knowledge [54] and less interested individuals may not have been motivated to participate. The study settings also introduce limitations as most the studies were from the U.S. healthcare system and examined inherited predisposition to cancer. No findings were unique to cancer; however, our findings may not be generalisable to other healthcare systems. The data provide a basis for hypotheses generation which can be prospectively tested in future studies.

Limitations arise from the systematic review methodology. As this is the first review to investigate clinician behaviour regarding genetic testing using the TDF, it was important to include studies with varied approaches to document the extent of published findings related to the topic. Thus, the heterogeneity of included studies precluded a meta-synthesis and we instead conducted a narrative analysis of findings from qualitative and quantitative studies which met quality standards. Finally, the role of medical 
specialists in relation to genetic testing was not always clearly articulated in the reviewed studies. It is possible that medical specialists in some studies would have to refer patients for testing, rather than being in a position to order tests themselves.

\section{Conclusions}

We sought to identify strategies which proved successful in the adoption of genetic testing to guide the introduction of genomics into clinical practice. Instead, we identified flaws in strategies widely espoused to support adoption of genomics by clinicians in mainstream healthcare. We find little evidence to support strong assertions of the value of education in changing clinician behaviour with respect to genetic or genomic tests. Education to build knowledge is an aspect of a clinician's capability, but this should not be relied on alone. We find the TDF provides a useful and comprehensive framework. The results of our study can now inform more nuanced interventions, such as organisational constraints, which may restrict clinicians' ability to offer genomic testing in the future, alongside those targeting factors intrinsic to the clinician.

Acknowledgements This study was supported by the Murdoch Children's Research Institute and the Victorian Government's Operational Infrastructure Support Program. The authors are grateful to Brenda Wilson for formative discussions and to Natalie Taylor for comments on the manuscript. The authors would like to acknowledge Margaret Sahhar and Jan Hodgson for supporting the original development of this research. All authors have no conflicts of interest to disclose.

\section{Compliance with ethical standards}

Conflict of interest The authors declare that they have no conflict of interest.

\section{References}

1. Weitzel KW, Alexander M, Bernhardt BA, et al. The IGNITE network: a model for genomic medicine implementation and research. BMC Med Genom. 2016;9:1.

2. Stark Z, Tan TY, Chong B, et al. A prospective evaluation of whole-exome sequencing as a first-tier molecular test in infants with suspected monogenic disorders. Genet Med. 2016;18: 1090-6.

3. Manolio TA, Abramowicz M, Al-Mulla F, et al. Global implementation of genomic medicine: we are not alone. Sci Transl Med. 2015;7:290ps213.

4. Directors ABo. Scope of practice: a statement of the American College of Medical Genetics and Genomics (ACMG). Genet Med. 2015;17:e3.

5. Addie S, Olson S, Beachy SH. Applying an implementation science approach to genomic medicine. Workshop SummaryRoundtable on translating genomic-based research for health; board on health sciences policy; health and medicine division; national academies of sciences, engineering, and medicine. Washington, DC: National Academies Press; 2016.

6. Feero WG, Green ED. Genomics education for health care professionals in the 21st century. J Am Med Assoc. 2011;306:989-90.

7. Khoury MJ, Berg A, Coates R, Evans J, Teutsch SM, Bradley LA. The evidence dilemma in genomic medicine. Health Aff. 2008;27:1600-11.

8. Rogowski WH, Grosse SD, Schmidtke J, Marckmann G. Criteria for fairly allocating scarce health-care resources to genetic tests: Which matter most. Eur J Hum Genet. 2014;22:25-31.

9. Brunham LR, Hayden MR. Medicine. Whole-genome sequencing: the new standard of care? Science. 2012;336:1112-3.

10. Gonzaga-Jauregui C, Lupski JR, Gibbs RA. Human genome sequencing in health and disease. Annu Rev Med. 2012;63:35-61.

11. Hastings R, de Wert G, Fowler B, et al. The changing landscape of genetic testing and its impact on clinical and laboratory services and research in Europe. Eur J Hum Genet. 2012;20:911-6.

12. Lerner-Ellis JP. The clinical implementation of whole genome sequencing: a conversation with seven scientific experts. J Inherit Metab Dis. 2012;35:689-93.

13. McCarthy JJ, McLeod HL, Ginsburg GS. Genomic medicine: a decade of successes, challenges, and opportunities. Sci Transl Med. 2013;5:189sr184.

14. Scheuner MT, Sieverding P, Shekelle PG. Delivery of genomic medicine for common chronic adult diseases: a systematic review. J Am Med Assoc. 2008;299:1320-34.

15. Chase GA, Geller G, Havstad SL, Holtzman NA, Bassett SS. Physicians' propensity to offer genetic testing for Alzheimer's disease: Results from a survey. Genet Med. 2002;4:297-303.

16. Van Riel E, Warlam-Rodenhuis C, Verhoef S, Rutgers E, Ausems M. BRCA testing of breast cancer patients: medical specialists' referral patterns, knowledge and attitudes to genetic testing. Eur J Cancer Care. 2010;19:369-76.

17. White DB, Bonham VL, Jenkins J, Stevens N, McBride CM. Too many referrals of low-risk women for BRCA1/2 genetic services by family physicians. Cancer Epidemiol Biomark Prev. 2008; 17:2980-6.

18. Hamilton AB, Oishi S, Yano EM, Gammage CE, Marshall NJ, Scheuner MT. Factors influencing organizational adoption and implementation of clinical genetic services. Genet Med. 2014;16:238-45.

19. Klitzman R, Chung W, Marder K, et al. Attitudes and practices among internists concerning genetic testing. J Genet Couns. 2013;22:90-100.

20. Registered Nurses Association of Ontario. Toolkit: implementation of clinical practice guidelines, 2002. http://rnao.ca/sites/rnaoca/files/BPG_Toolkit_0.pdf.

21. Brand CA, Barker AL, Morello RT, et al. A review of hospital characteristics associated with improved performance. Int J Qual Health Care. 2012;24:483-94.

22. Scott I. What are the most effective strategies for improving quality and safety of health care? Intern Med J. 2009;39:389-400.

23. Roberts MC, Kennedy AE, Chambers DA, Khoury MJ. The current state of implementation science in genomic medicine: opportunities for improvement. Genet Med. 2017;19:858-63.

24. Department of Health. Annual report of the Chief Medical Officer 2016: generation genome, 2017. https://www.gov.uk/government/ uploads/system/uploads/attachment_data/file/631043/CMO_a nnual_report_generation_genome.pdf.

25. Cane J, O'Connor D, Michie S. Validation of the theoretical domains framework for use in behaviour change and implementation research. Implement Sci. 2012;7:37-53.

26. Michie S, van Stralen MM, West R. The behaviour change wheel: a new method for characterising and designing behaviour change interventions. Implement Sci. 2011;6:42-53. 
27. Moher D, Liberati A, Tetzlaff J, Altman DG. The PG: preferred reporting items for systematic reviews and meta-analyses: the PRISMA statement. PLoS Med. 2009;6:e1000097.

28. Popay J, Roberts H, Sowden A et al. Guidance on the conduct of narrative synthesis in systematic reviews: a product from the ESRC methods programme. Lancaster:Lancaster University; 2006. http://citeseerx.ist.psu.edu/viewdoc/download?doi=10.1.1. $178.3100 \&$ rep $=$ rep $1 \&$ type $=$ pdf

29. Elo S, Kyngas H. The qualitative content analysis process. J Adv Nurs. 2008;62:107-15.

30. QSR International. NVivo qualitative data analysis Software, Version 10; Melbourne, VIC:QSR International Pty Ltd.; 2012.

31. Kmet LM, Lee RC, Cook LS. Standard quality assessment criteria for evaluating primary research papers from a variety of fields. Edmonton: Alberta Heritage Foundation for Medical Research; 2004.

32. Paul J, Metcalfe S, Stirling L, Wilson B, Hodgson J. Analyzing communication in genetic consultations-a systematic review. Patient Educ Couns. 2015;98:15-33.

33. Acharya K, Schindler A. Developmental and behavioral pediatricians' attitudes toward screening for fragile X. Am J Intellect Dev Disabil. 2013;118:284-93.

34. Beitsch PD, Whitworth PW. Can breast surgeons provide breast cancer genetic testing? An American Society of Breast Surgeons survey. Ann Surg Oncol. 2014;21:4104-8.

35. Birmingham WC, Agarwal N, Kohlmann W, et al. Patient and provider attitudes toward genomic testing for prostate cancer susceptibility: a mixed method study. BMC Health Serv Res. 2013;13:279.

36. Carroll JC, Brown JB, Blaine S, Glendon G, Pugh P, Medved W. Genetic susceptibility to cancer. Family physicians' experience. Cancer Fam Physician. 2003;49:45-52.

37. Corkindale D, Ward H, McKinnon R. Low adoption of pharmacogenetic testing: an exploration and explanation of the reasons in Australia. Pers Med. 2007;4:191-9.

38. Cox SL, Zlot AI, Silvey K, et al. Patterns of cancer genetic testing: a randomized survey of Oregon clinicians. J Cancer Epidemiol. 2012;2012:1-11.

39. Darcy D, Tian L, Taylor J, Schrijver I. Cystic fibrosis carrier screening in obstetric clinical practice: knowledge, practices, and barriers, a decade after publication of screening guidelines. Genet Test Mol Biomark. 2011;15:517-23.

40. Doksum T, Bernhardt BA, Holtzman NA. Carrier screening for cystic fibrosis among Maryland obstetricians before and after the 1997 NIH Consensus Conference. Genet Test. 2001;5:111-6.

41. Doksum T, Bernhardt BA, Holtzman NA. Does knowledge about the genetics of breast cancer differ between nongeneticist physicians who do or do not discuss or order BRCA testing? Genet Med. 2003;5:99-105.

42. Freedman AN, Wideroff L, Olson L, et al. US physicians' attitudes toward genetic testing for cancer susceptibility. Am J Med Genet A. 2003;120A:63-71.

43. Geller G, Bernhardt BA, Doksum T, Helzlsouer KJ, Wilcox P, Holzman NA. Decision-making about breast cancer susceptibility testing: how similar are the attitudes of physicians, nurse practitioners, and at-risk women? J Clin Oncol. 1998;16:2868-76.

44. Harris BU, Miyake CY, Motonaga KS, Dubin AM. Diagnosis and management of pediatric Brugada syndrome: a survey of pediatric electrophysiologists. Pacing Clin Electrophysiol. 2014;37:638-42.

45. James C, Geller G, Bernhardt BA, Docksum T, Holtzman NA. Are practicing and future physicians prepared to obtain informed consent? The case of genetic testing for susceptibility to breast cancer. Community Genet. 1998;1:203-12.

46. Kadafour M, Haugh R, Posin M, Kayser SR, Shin J. Survey on warfarin pharmacogenetic testing among anticoagulation providers. Pharmacogenomics. 2009;10:1853-60.
47. Klitzman R, Abbate KJ, Chung WK, et al. Psychiatrists' views of the genetic bases of mental disorders and behavioral traits and their use of genetic tests. J Nerv Ment Dis. 2014;202:530-8.

48. Lubin IM, McGovern MM, Gibson Z, et al. Clinician perspectives about molecular genetic testing for heritable conditions and development of a clinician-friendly laboratory report. J Mol Diagn. 2009;11:162-71.

49. McGowan ML, Fishman JR, Settersten RA, Lambrix MA, Juengst ET. Gatekeepers or intermediaries? The role of clinicians in commercial genomic testing. PLoS ONE. 2014;9: e108484.

50. Myers MF, Chang M-H, Jorgensen C, et al. Genetic testing for susceptibility to breast and ovarian cancer: evaluating the impact of a direct-to-consumer marketing campaign on physicians' knowledge and practices. Genet Med. 2006;8:361-70.

51. Najafzadeh M, Davis JC, Joshi P, Marra C. Barriers for integrating personalized medicine into clinical practice: a qualitative analysis. Am J Med Genet A. 2013;161A:758-63.

52. Ngoi N, Lee SC, Hartman M, Khin LW, Wong A. Interest and attitudes of patients, cancer physicians, medical students and cancer researchers towards a spectrum of genetic tests relevant to breast cancer patients. Breast. 2013;22:47-52.

53. Nyrhinen T, Hietala M, Puukka P, Leino-Kilpi H. Privacy and equality in diagnostic genetic testing. Nurs Ethics. 2007;14:295-308.

54. Nyrhinen T, Leino-Kilpi H, Hietala M. Ethical issues in the diagnostic genetic testing process. New Genet. 2004; 23:73-87.

55. Pal T, Cragun D, Lewis C, et al. A statewide survey of practitioners to assess knowledge and clinical practices regarding hereditary breast and ovarian cancer. Genet Test Mol Biomark. 2013;17:367-75.

56. Peppercorn J, Hamilton E, Marcom PK, Beskow L, Lyman GH. Pharmacogenetic testing in the face of unclear clinical efficacy: lessons from cytochrome P450 2D6 for tamoxifen. Cancer. 2013;119:3703-9.

57. Plon SE, Cooper HP, Parks B, et al. Genetic testing and cancer risk management recommendations by physicians for at-risk relatives. Genet Med. 2011;13:148-54.

58. Reiff M, Mueller R, Mulchandani S, Spinner N, Pyeritz R, Bernhardt B. A Qualitative Study of Healthcare Providers' Perspectives on the implications of genome-wide testing in pediatric clinical practice. J Genet Couns. 2014;23:474-88.

59. Salm M, Abbate K, Appelbaum P, et al. Use of genetic tests among neurologists and psychiatrists: knowledge, attitudes, behaviors, and needs for training. $\mathrm{J}$ Genet Couns. 2014;23:156-63.

60. Selkirk CG, Weissman SM, Anderson A, Hulick PJ. Physicians' preparedness for integration of genomic and pharmacogenetic testing into practice within a major healthcare system. Genet Test Mol Biomark. 2013;17:219-25.

61. Stanek EJ, Sanders CL, Taber KAJ, et al. Adoption of pharmacogenomic testing by US physicians: results of a nationwide survey. Clin Pharmacol Ther. 2012;91:450-8.

62. Vadaparampil ST, Wideroff L, Olson L, Viswanath K, Freedman AN. Physician exposure to and attitudes toward advertisements for genetic tests for inherited cancer susceptibility. Am J Med Genet A. 2005;135:41-46.

63. Van Der Zwaag AM, Weinreich SS, Bosma AR, et al. Current and best practices of genetic testing for maturity onset diabetes of the young: views of professional experts. Public Health Genomics. 2014;18:52-59.

64. Walden LM, Brandl EJ, Changasi A, et al. Physicians' opinions following pharmacogenetic testing for psychotropic medication. Psychiatry Res. 2015;229:913-8. 
65. Walsh J, Arora M, Hosenfeld C, Ladabaum U, Kuppermann M, Knight SJ. Preferences for genetic testing to identify hereditary colorectal cancer: perspectives of high-risk patients, community members, and clinicians. J Cancer Educ. 2012;27:112-9.

66. Wideroff L, Freedman AN, Olson L, et al. Physician use of genetic testing for cancer susceptibility: results of a national survey. Cancer Epidemiol Biomark Prev. 2003;12:295-303.

67. Wilkins-Haug L, Erickson K, Hill L, Power M, Holzman GB, Schulkin J. Obstetrician-gynecologists' opinions and attitudes on the role of genetics in women's health. J Women Health Gend Based Med. 2000;9:873-9.

68. Wilkins-Haug L, Hill LD, Power ML, Holzman GB, Schulkin J. Gynecologists' training, knowledge, and experiences in genetics: a survey. Obstet Gynecol. 2000;95:421-4.

69. Will CM, Armstrong D, Marteau TM. Genetic unexceptionalism: clinician accounts of genetic testing for familial hypercholesterolaemia. Soc Sci Med. 2010;71:910-7.
70. Flottorp SA, Oxman AD, Krause J, et al. A checklist for identifying determinants of practice: a systematic review and synthesis of frameworks and taxonomies of factors that prevent or enable improvements in healthcare professional practice. Implement Sci. 2013;8:35

71. Rainbird K, Sanson-Fisher R, Buchan H. Identifying barriers to evidence uptake. Melbourne: National Insitute of ClinicalStudies; 2006. https://www.nhmrc.gov.au/_files_nhmrc/publications/atta chments/nic55_identifying_barriers_to_evidence_uptake_150720. pdf

72. Légaré $F$, Robitaille $H$, Gane $C$, Hébert J, Labrecque $M$, Rousseau F. Improving decision making about genetic testing in the clinic: an overview of effective knowledge translation interventions. PLoS ONE. 2016;11:e0150123.

73. Vassy JL, Christensen KD, Schonman EF, et al. The impact of whole-genome sequencing on the primary care and outcomes of healthy adult patients: a pilot randomized trial. Ann Intern Med. 2017;167:159-69. 